\title{
What are the Factors and the Method Used for the Formation of Nasal Septal Perforations due to Septoplasty?
}

\author{
Erkan YILDIZ* \\ Department of Anatomy, Harran University Faculty of Medicine, Turkey
}

*Corresponding author: Erkan YILDIZ, Department of Anatomy, Harran University Faculty of Medicine, Turkey

\begin{abstract}
Objective: Nasal septal perforation is the most common complication in the long term after septoplasty. Nasal septal perforation is the partial opening of the wall between both nasal cavities. In this study, we will evaluate the causes of septal perforation after septoplasty.

Materials and methods: 400 patients aged between 18 and 50 years who underwent septoplasty operation in Afyonkarahisar Şuhut State Hospital between 2016-2019 were evaluated retrospectively. These patients were divided into groups with and without perforation. Smoking and alcohol use of patients with perforation, gender and septum stabilization was used for the technique.

Results: There were 280 male and 120 female patients. 378 patients had no postoperative septal perforation. 22 had perforation after surgery. Of the patients in the perforated group, 19 male and 3 female were female. 21 of these patients smoked, 1 patient did not drink. 11 of these patients were drinking alcohol and 10 of them did not drink alcohol. Transseptal suturation + nasal saturation splint was used in 14 patients to stabilize the septum while perforation rate was $22 / 400(1.3 \%)$. Male sex ratio was significantly dominant in perforated patients. $(19 / 3)(p<0.05)$ Smoking group was significantly increased in patients with perforated group $(21 / 1)(p<0.05)$ There was no significant difference in perforation rate and alcohol intake among the patients in this group $(p<0.05)$. A significant difference was observed between the methods used to stabilize the septum $(\mathrm{P}<$ $0.05)$. The perforation rate was significantly increased in transseptal sutures.
\end{abstract}

Conclusion: Patients with perforation after septoplasty were found to increase the risk of perforation by male sex, smoking and surgical technique as transseptal suturing technique.

\section{Keywords}

Nasal septal perforation, Septoplasty, Suturation, Smoking

\section{Introduction}

Nasal septal perforation; The nasal septum consists of the cartilage, the bone septum and the mucosal layer covering it. The bone septum consists of the vomer, the lamina perpendicular of the ethmoid bone and the maxillary crest. As a result of necrosis of the septum due to damage to these layers, there is a gap between the two nasal cavities. Therefore, the nasal airflow is disturbed and leads to deterioration in the quality of life of the patient [1].

Although the incidence of septal perforation is reported to be around $1 \%$, it is actually much more. Septal perforations may occur due to iatrogenic, trauma, drug use (steroids, cocaine, etc.) and cauterization. The most common cause of septum surgery is secondary to infection.

The most common site of formation of septum perforations is the anterior region. The posterior or superior source is about $10 \%$. Perforations in the anterior region lead to clinical symptoms, and posteriordal patients do not produce much clinical signs. The most common cause of these patients is nosebleed, nasal congestion and nasal dryness. Rarely, there are complaints of sound extraction similar to whistling [1,2].

In the treatment, first of all, conservative method is observed. If the patient's complaints persist, surgical treatment is used. Topical washing, moisturizing and antibiotic creams are applied to the patient. Nasal septal buttons and endoscopic approaches are used for surgical treatment. Several flap techniques have been reported in relation to endoscopic approaches [3-5]. 


\section{Materials and Methods}

400 patients aged between 18 and 50 years who underwent septoplasty operation in Afyonkarahisar Şuhut State Hospital between 2016-2019 were evaluated retrospectively. These patients consisted of patients with septoplasty due to nasal septal deviation. All patients underwent septoplasty with closed technique. In some patients, transseptal suture technique was used at the end of septoplasty. In the transseptal

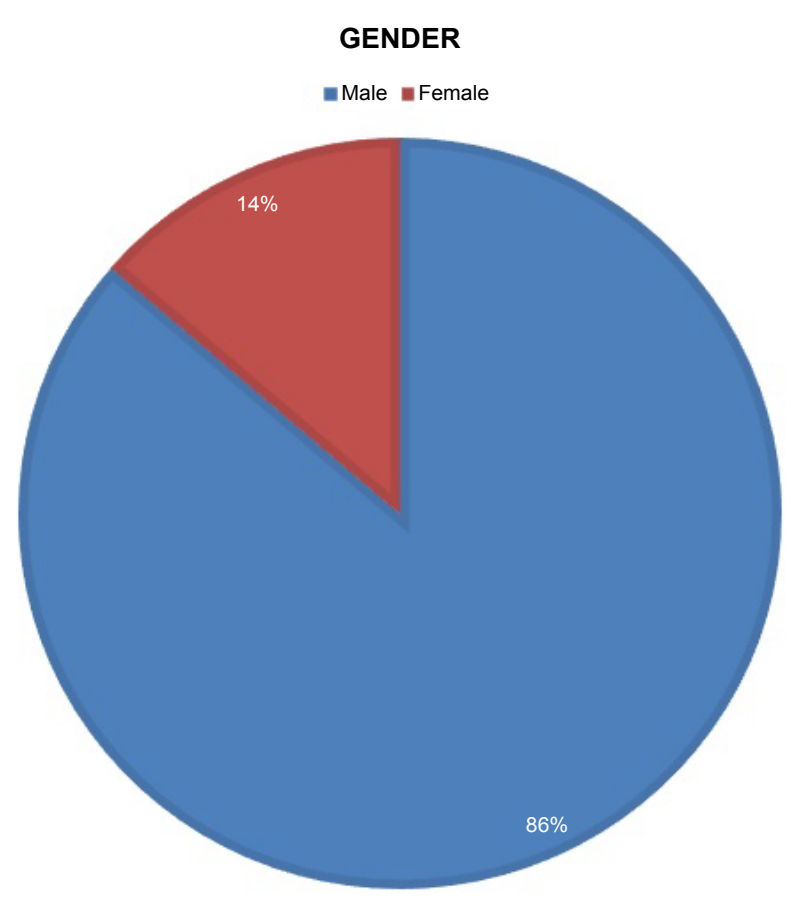

Figure 1: Comparing nasal septal perforation by gender.

\section{SMOKING}

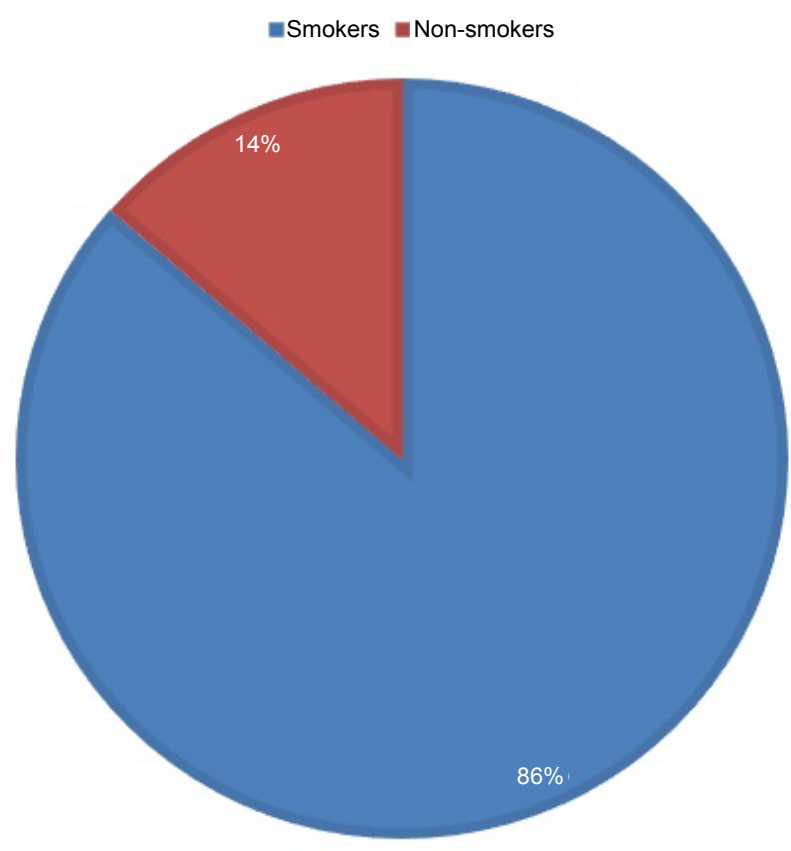

Figure 2: Comparison of smoking and non-smokers septal perforation. suturation, 4.0 rapid Vicril was used. Nasal splints were used for all patients. These patients were divided into two groups with and without perforation. Demographic characteristics of the patients were recorded. The smoking and alcohol use of the patients with perforation were evaluated in terms of the technique used for septum stabilization. Consent was obtained from the ethics committee of Afyon Kocatepe University Faculty of Medicine [3].

\section{Statistical Analysis}

SPSS 21.0 software (IBM, SPSS, Chicago, USA) was used for analysis. The categorical data were given as number $(\mathrm{n})$ and percentage (\%). Chi-square test and Mann-Whitney $U$ test were used to compare the characteristics of the data, smoking-alcohol use and the surgical method used. $\mathrm{P}<0.05$ was considered statistically significant.

\section{Results}

There were 280 male and 120 female. 378 patients had no postoperative septal perforation. 22 had perforation after surgery. Of the patients in the perforated group, 19 male and 3 female were female. Male sex ratio was significantly dominant in perforated patients. $(19 / 3)(p<0.05)$ (Figure 1) 21 of these patients were smoking and 1 patient was not drinking (Figure 2). 11 of these patients were drinking alcohol and 10 of them did not drink alcohol (Figure 3). To stabilize the septum in patients with perforation While transseptal suturation + nasal saturation splint was used in 14 patients, only nasal saturation splint was used in 8 patients (Figure 4). The perforation rate after surgery was $22 / 400$ $(1.3 \%) .(21 / 1)(p<0.05)$ There was no significant difference in the perforation rate among the patients in this group and in those with alcohol intake. $(p>0.05)$ There was no significant difference between the methods used to stabilize the septum $(p<0.05)$. The rate of perforation was significantly increased in patients who underwent transseptal suturation.

\section{Discussion}

The septum is the septic cartilage that separates the nasal cavity consisting of the bone at the back and the mucosa (mucoperikondrium, mukoperiostium) surrounding it. By providing nasal airflow from front to back, health provides a breath function. In septal perforations, this layer is primarily infected due to iatrogenic or surgical trauma, and then mucosal blood flow is impaired. Over time, small openings grow in this layer. Patients present with complaints such as nasal congestion, nasal bleeding, drying and crusting, whistling, and quality of life. granulomatous diseases, topical drug use (steroids and cocaine), bilateral nasal cauterization, nasal tampons, nose piercings. They are the most common anterior and rarely originate posterior and superior $[6,7]$.

Septal perforation surgery is quite difficult. Con- 


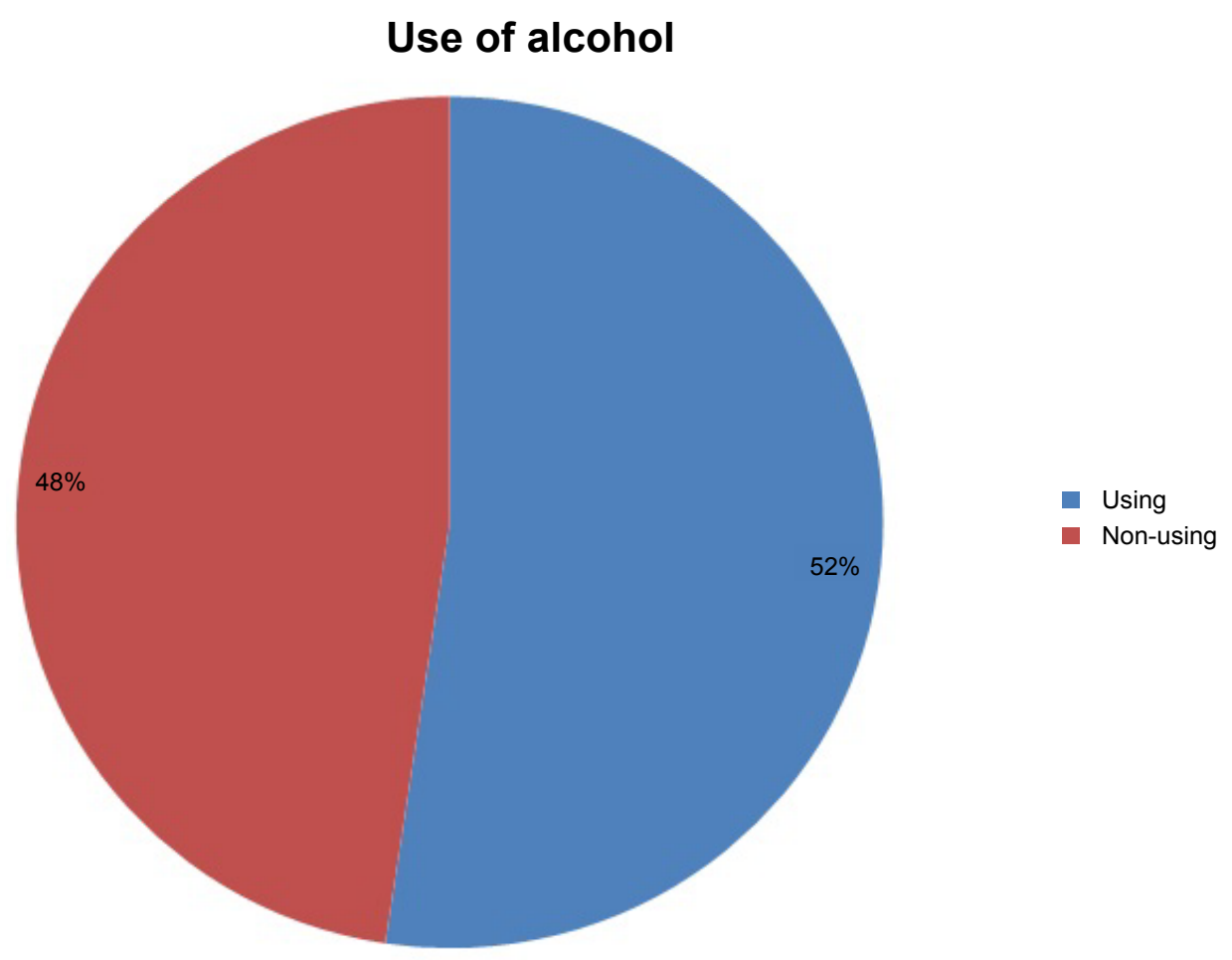

Figure 3: The comparison in terms of perforation with those who use alcohol users.

\section{SEPTUM STABILIZATION TECHNIQUE}

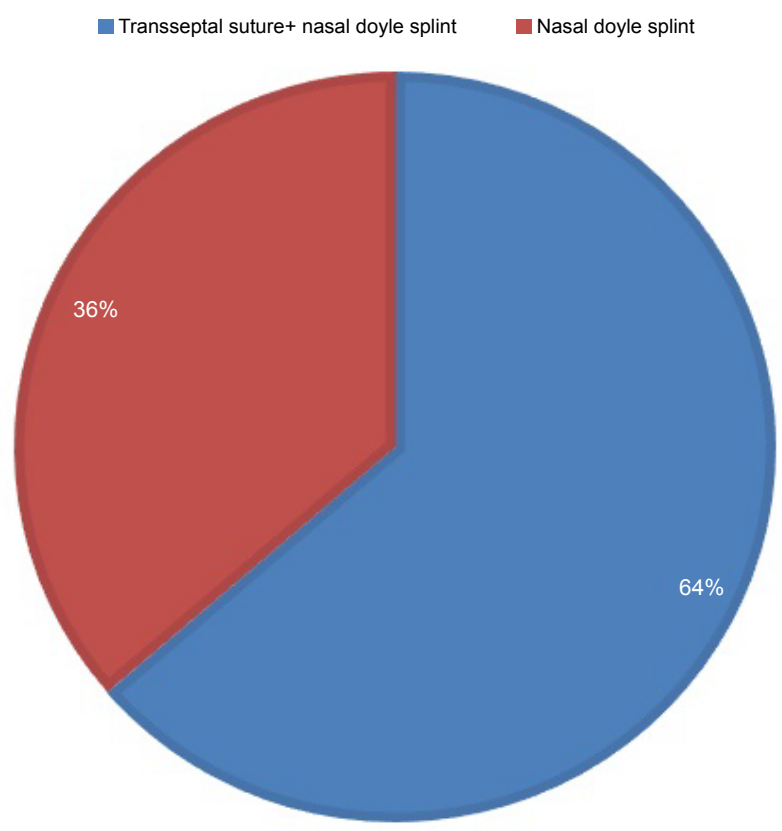

Figure 4: Comparison of septum stabilization technique.

servative approach is recommended in the treatment. Vaseline moisturizing ointments, antibiotic ointments, postnasal drainage are the most common. Surgical treatment is used for patients who cannot relax with these methods. The most commonly used method was the nasal septal button method, and now the endoscopic flap translation method is the most common and successful treatment method. Although the nasal septal buttons have symptoms in a short time, they are not very successful in the long term. Flap methods, the success of experienced surgical hands is very high [810]. Generally extracorporeal technique is used in septoplasty repair [11].

Smoking and alcohol use are not required in patients undergoing nasal septum surgery. They have a negative effect on wound healing [12]. In a study conducted by Yazici ZM, et al. Cigarette smoking has been shown to decrease postoperative quality of life in patients undergoing septoplasty [13]. In another study, it was observed that smoking increased the risk of perforation [14]. Increased suturing after septum surgery leads to crusting, causing infection and mucosal damage in that area of the septum [15]. Therefore, suturation techniques for reducing crusting in septum surgery have been described. In addition, vaseline moisturizers and nasal washing are recommended. In the study on respiratory stress and complication of tamponade and septal suturation, the use of merosel buffer and smoking increased this risk [16]. In another study, no significant difference was observed between the use of tamponade and septal perforation relationship [17].

There is not much literature on the etiology of septum perforation. There are several studies suggesting that smoking increases perforation [18]. The effect of sex, alcohol use, surgical tamponade or suturation with perforation has not been investigated. In our study, a significant relationship was found between male sex and smoking and septal perforation. No significant increased risk was found in alcohol use. In the stabilization of septoplasty, the risk of septal perforation was not increased in the use of nasal tamponade alone, but 
the risk of septal perforation was increased in transeptal sutures.

In conclusion, in septum surgery, smoking and transseptal suturation technique are risk factors for septal perforation. There was no significant increase in alcohol use.

\section{References}

1. Lanier B, Kai G, Marple B, Wall GM (2007) Pathophysiology and progression of nasal septal perforation. Annals of Allergy, Asthma \& Immunology 99: 473-480.

2. Pereira C, Santamaría A, Langdon C, López-Chacón M, Hernández-Rodríguez J, et al. (2018) Nasoseptal Perforation: from Etiology toTreatment. Current Allergy and Asthma Reports 18: 5.

3. Tastan E, Aydogan F, Aydin E, Can IH, Demirci M, et al (2012) Inferior turbinate composite graft for repair of nasal septal perforation. Am J Rhinol Allergy 26: 237-242.

4. Kaya E, Cingi C, Olgun Y, Soken H, Pinarbasli Ö (2015) Three layer interlocking: A novel technique for repairing a nasal septum perforation. Ann Otol Rhinol Laryngol 124: 212-215.

5. Cassano M (2017) Endoscopic repair of nasal septal perforation. Acta Otorhinolaryngologica Italica 37: 486-492.

6. Lumsden A, Shakeel M, Ah-See KL, Supriya M, Ah-See $\mathrm{KW}$, et al. (2015) Management of nasal septal perforation: Grampian experience. Austin J Otolaryngol 2: 1041.

7. Sapmaz E, Toplu Y, Somuk BT (2018) A new classification for septal perforation and effects of treatment methods on quality of life. Brazilian Journal of Otorhinolaryngology.

8. Kridel, Russell WH (2004) Considerations in the etiology, treatment, and repair of septal perforations. Facial Plastic Surgery Clinics 12: 435-450.

9. Ribeiro JS, da Silva GS (2007) Technical advances in the correction of septal perforation associated with closed rhinoplasty. Archives of Facial Plastic Surgery 9: 321-327.
10. Re M, Paolucci L, Romeo R, Mallardi V (2006) Surgical treatment of nasal septal perforations. Our experience. Acta Otorhinolaryngologica Italica 26: 102-109.

11. Bohluli B, Mohammad Hosein Kalantar Motamedi, Payam Varedi, Mansour Malekzadeh, Alireza Ghassemi, et al. (2014) Management of perforations of the nasal septum: Can extracorporeal septoplasty be an effective option? Journal of Oral and Maxillofacial Surgery 72: 391395.

12. Ozdemir S, Celik H, Cengiz C, Zeybek ND, Bahador E, et al. (2019) Histopathological effects of septoplasty techniques on nasal septum mucosa: An experimental study. European Archives of Oto-Rhino-Laryngology 276: 421-427.

13. Yazici ZM, Sayin I, Erdim I, Gunes S, Kayhan FT (2015) The effect of tobacco smoking on septoplasty outcomes: A prospective controlled study. Hippokratia 19: 219-224.

14. Cetiner H, Cavusoglu I, Duzer S (2017) The effect of smoking on perforation development and healing after septoplasty. American Journal of Rhinology \& Allergy 31: 63-65.

15. Dadgarnia M, Meybodian M, Karbasi A, Baradaranfar M, Atighechi S, et al. (2017) Comparing nasal packing with trans-septal suturing following septoplasty: A randomized clinical trial. Eur Arch Otorhinolaryngol 274: 3513-3518.

16. Kayahan B, Ozer S, Suslu AE, Ogretmenoglu O, Onerci M (2017) The comparison of the quality of life and intranasal edema between the patients with or without nasal packing after septoplasty. Eur Arch Otorhinolaryngol 274: 15511555.

17. Deniz M, Çiftçi Z, Işık A, Demirel OB, Gültekin E (2014) The impact of different nasal packings on postoperative complications. American Journal of Otolaryngology 35: 554-557.

18. Chen PG, Floreani S, Wormald PJ (2018) The utility of enlarging symptomatic nasal septal perforations. Ear Nose Throat J 97: E41-E43. 